\title{
ARMADILLO : ENTRE IMAGES DE GUERRE CONTEMPORAINES ET MISE EN SCÈNE DU RÉEL
}

\author{
Lise HENRIC ${ }^{1}$
}

Face à la multiplication des images de guerre que ce soit au cinéma, à la télévision ou encore sur les réseaux sociaux numériques, il nous appartient de nous interroger sur la diffusion de ces images contemporaines afin de mieux appréhender la transcendance du réel ; prétention à l'égard de la question de l'authenticité et du spectateur (Picard, 2018) ou nouvelle forme d'adhérence au réel ?

Permettant d'actualiser notre réflexion sur la relation complexe du cinéma au réel, nous nous attacherons à discuter de l'ambiguïté de certaines images contemporaines de la guerre en nous appuyant sur le film Armadillo (Metz, 2010). Considéré comme un documentaire par certains médias et comme une fiction par plusieurs critiques de cinéma, Armadillo (nom d'une caserne en Afghanistan tenue par des soldats danois) suit le quotidien de nouvelles recrues durant les six mois de leur présence dans le camp. Réalisé par Janus Metz, Armadillo a été présenté à la Semaine de la Critique, durant le festival de Cannes, où il a remporté le Grand Prix de la semaine internationale de la critique en 2010 .

À travers l'étude de ce film, il convient donc de nous questionner sur la frontière ténue entre fiction et réel et envisager le film de guerre dans toute sa complexité. Quel rapport au réel le film Armadillo impliquet-il chez le spectateur? Quelles sont les répercussions de ces images? Peut-on proposer une pédagogie de l'image qui soit efficace ? Comment interpréter les images saisies par un réalisateur surtout quand le genre cinématographique n'est pas clairement défini ?

1 Lise Henric est docteure en Sciences de l'Information et de la Communication. Elle est actuellement chercheure-associée au laboratoire CEREGE (Université de Poitiers).

Recherches en communication, $\mathrm{n}^{\circ} 50$ - Article publié le 11/08/2020 


\section{Introduction}

Face à la multiplication des images de guerre que ce soit au cinéma, à la télévision ou encore sur les réseaux sociaux numériques, il nous appartient de nous interroger sur la diffusion de ces images contemporaines. Si l'on peut soutenir à la fois que « tout film est un film de fiction » (Metz, 1977) et que «tout film est un documentaire sur son propre tournage » (Rivette, 1950) c'est bien que la frontière entre fiction et réel est de plus en plus ténue. Cette approche souligne le besoin d'ouvrir une discussion sur la nécessité de définir les outils liés au déchiffrage des images.

Le film Armadillo (Metz, 2010), sorti en salles françaises le 15 décembre 2010, suit le quotidien de nouvelles recrues danoises durant les six mois de leur présence dans un camp militaire situé en Afghanistan, entre leurs discussions avec les civils locaux, leurs moments de détente et leurs actions militaires contre les talibans. Réalisé par Janus Metz ${ }^{3}$, Armadillo a été présenté à la Semaine de la Critique, durant le festival de Cannes, où il a remporté le Grand Prix de la semaine internationale de la critique en 2010. Selon le bilan $2010 \mathrm{du}$ CNC publié en mai 2011, les films documentaires représentent 2,6\% des entrées en France. À titre de comparaison, selon CBO Box-Office, Armadillo a cumulé 7.388 entrées durant la première semaine de sa sortie (du 15 au 21 décembre 2010) tandis que le documentaire Océans (Perrin, 2010) cumule 774.586 entrées.

Axant nos recherches sur le thème des docufictions à la télévision, genre difficile à définir et au contour flou en raison de la frontière ténue entre fiction et réel, notre article nous permet d'approfondir nos observations dans une dynamique de réflexion pluridisciplinaire. L'étude de ce film permet-elle à certains cinéphiles, chercheurs, critiques et membres de la profession de poser un regard critique sur la construction des images au cinéma ? Peut-on parler d'une transcendance du réel, accentuée par l'usurpation du réel, de ses codes visuels, tout en dénouant l'écheveau complexe liant fiction et documentaire ou « convient-il de rendre le réel à lui-même, à l'insignifiance » (Rosset, 1978) ?

2 Nom d'une caserne en Afghanistan tenue par des soldats danois.

3 Né en 1974 au Danemark, Janus Metz devient célèbre en 2008 avec deux films : Love on Delivery et Ticket to Paradise. Il a réalisé également Borg/McEnroe centré sur la relation et la rivalité entre Bjorn Borg et John McEnroe dans le cadre du tournoi de Wimbledon. 
Comment définir Armadillo? Fiction, documentaire ou docufiction?

Le genre comme catégorie de réception est conçu comme une promesse qui est spécifiée par le type de flux, par un mode énonciatif et par un ton. Promesse d'une relation à un monde dont le mode ou le degré d'existence conditionne l'adhésion ou la participation du spectateur en fonction de savoir, de croyances et d'émotions (Jost, 1997).

Comment les médias et la presse française, en particulier, ont-ils catégorisé le film Armadillo? Si nous nous référons aux propos suivants, Armadillo est considéré comme un documentaire. Pour Léo Soesanto, «La Semaine de la Critique n'avait jamais accueilli de documentaire depuis qu'elle était devenue compétitive en $1990^{4} »$. Le Point, hebdomadaire d'actualité, quant à lui titre : «Armadillo, la guerre en vrai. Documentaire ${ }^{5} »$. France Inter souligne : «Armadillo, le piège afghan, un documentaire inédit sur Arte $»^{6}$. En ce sens, les médias, en utilisant le terme « documentaire » soulignent l'aspect authentique de ce film.

D'autres critiques, dont Éric Libiot, émettent des doutes : «Fiction ou réalité ? Je n'en savais rien à l'époque et cette incertitude ajoutait à ce léger flottement, évidemment voulu par le réalisateur, qui place le spectateur non seulement au cœur d'un conflit - et, là, ce n'est pas une formule - mais aussi face à la puissance d'images vraies mais dramatisées qui tirent Armadillo vers un genre hybride, tendant à remplacer le reportage télé, devenu irréel - une guerre, c'est loin et c'est les autres par un documentaire suffisamment romanesque pour qu'il provoque un puissant effet de réel ${ }^{7} \gg$. Jean-Michel Frodon, historien du cinéma, sou-

4 Portrait du film comme un documentaire par Léo Soesento, journaliste français freelance. Accès : http://archives.semainedelacritique.com/films/2010/2010_comp_armadi.php. [Consulté le 23/04/2018].

5 Présentation du film Armadillo comme un documentaire publié sur le site internet du Point le 13/12/10. Accès : http://www.lepoint.fr/cinema/armadillo-la-guerre-envrai-13-12-2010-1274613_35.php. [Consulté le 23/04/2018].

6 Lien vers l'émission de France Inter « Le cabinet de curiosités » présentant $A r$ madillo comme un documentaire. Accès : https://www.franceinter.fr/emissions/ le-cabinet-de-curiosites/le-cabinet-de-curiosites-05-decembre-2012. [Consulté le 23/04/2018].

7 Critique par Éric Libiot du film Armadillo sur le site de l'Express le 15/12/10. Accès : https://www.lexpress.fr/culture/cinema/armadillo-du-lard-ou-de-la-guerre_945208. html. [Consulté le 23/04/2018]. 
ligne : «Que le film ait été présenté, et primé, à Cannes dans la sélection La Semaine de la critique sans que soit plus interrogée sa mise en scène et les questions complexes qu'elle soulève, laisse perplexe ${ }^{8} »$.

Plus largement, la présentation de notre étude de cas nous permet de discuter de l'ambiguïté de certaines images contemporaines de la guerre et de nous interroger sur « la nature ontologique du $7^{\mathrm{e}}$ art qui renvoie à une certaine conception du monde » (Frodon, 1998). En effet, si les images sont le témoignage oculaire tout au long de l'histoire, quelles portées ont donc ces images sur le spectateur? Où commence et où finit la fiction ? Peut-on justifier, par conséquent, de l'utilisation du terme hybridation, pour le film Armadillo?

Ces questionnements rejoindront le point de départ à l'origine de nos recherches et de nos objets d'étude.

\section{Les questions du sujet, de la création, de la production et de la diffusion}

Dès la bande annonce, le réalisateur présente le film Armadillo de la façon suivante : "Ce n'est pas une fiction, ce n'est pas un film de guerre, juste... la réalité ». Nous sommes donc face à des images qui sont présentées par leur auteur comme réalistes. Quel est donc le rapport qu'entretient un réalisateur avec les images de guerre?

« Cette tendance contemporaine liée à la pensée post-moderne, à la pensée déconstructiviste consiste à refuser de tracer une frontière nette entre fiction et non-fiction » (Lioult, 2010) ${ }^{9}$. Dans ce contexte et face à ce trouble contemporain, il convient de proposer une grille de lecture afin de travailler théoriquement la différence entre documentaire et fiction en nous focalisant sur notre étude de cas. En effet, en énonçant les divers éléments pouvant indiquer aux spectateurs qu'ils sont face à un documentaire ou à une fiction, nous pourrons ainsi faire apparaître les nouvelles exigences spécifiques au genre proposées par

8 Dans cet article publié en 2010, Jean-Michel Frodon s'interroge sur les moyens par lesquels le cinéma construit les images. Accès : http://www.slate.fr/story/31837/ documentaire-art-fiction. [Consulté le 23/04/2018].

9 Dans cette vidéo-cours, Jean-Luc Lioult propose sa définition du film documentaire et s'interroge sur l'ambivalence des images du réel. Accès : https:/www.canalu. tv/video/tcp_universite_de_provence/tentatives_de_definitions_du_film_documentaire_penser_le_cinema_documentaire_lecon_2.6943. [Consulté le 27/04/2018]. 
le film Armadillo et tenter de délimiter cette frontière ténue entre fiction et documentaire.

Tableau 1. Recensement des éléments de marquage indiciel

\begin{tabular}{|c|c|}
\hline $\begin{array}{c}\text { Éléments de marquage indiciel } \\
\text { de la fiction }\end{array}$ & $\begin{array}{c}\text { Éléments de marquage indiciel } \\
\text { du documentaire }\end{array}$ \\
\hline $\begin{array}{l}\text { Armadillo débute par une situation tem- } \\
\text { porelle : le mois de Janvier, mois durant } \\
\text { lequel les événements se produisent, } \\
\text { mais sans nous préciser l'année. Tout } \\
\text { document historique et archives sont nor- } \\
\text { malement datés. }\end{array}$ & $\begin{array}{l}\text { Rôle des soldats danois tenus par les sol- } \\
\text { dats danois eux-mêmes (pas d'acteurs). }\end{array}$ \\
\hline $\begin{array}{l}\text { Place prépondérante à la musique accen- } \\
\text { tuant le côté dramaturgique de certaines } \\
\text { scènes. }\end{array}$ & $\begin{array}{l}\text { Approche des effets de réel en s'inspirant } \\
\text { des émissions de télé-réalité : le specta- } \\
\text { teur entend la voix du commandant don- } \\
\text { nant des ordres aux soldats mais sans le } \\
\text { voir à la caméra ; référence à la voix-off } \\
\text { donnant des missions aux candidats. Inti- } \\
\text { mité des personnages. }\end{array}$ \\
\hline $\begin{array}{l}\text { Certaines images sont prises à partir des } \\
\text { casques des soldats ou en caméra portée } \\
\text { par le cadreur (images tremblantes, ins- } \\
\text { tables et des plans mal cadrés). Sensa- } \\
\text { tion d'image vérité-réalité permettant de } \\
\text { générer des effets de réel ; figure majeure } \\
\text { de notre contemporanéité comme dans } \\
\text { certaines fictions telles que le Projet } \\
\text { Blair Witch (Myrick, Sanchez, 1999). }\end{array}$ & $\begin{array}{l}\text { Utilisation de nombreux titrages dès les } \\
\text { premières minutes du film : « Helmand } \\
\text { Province, Southern Afghanistan, } 2009 \text {, } \\
\text { « Forward Operation, Base Armadillo». }\end{array}$ \\
\hline $\begin{array}{l}\text { Présence au générique d'une directrice } \\
\text { de casting : Camilla Majid. } \\
\text { Présence au générique d'un conseiller } \\
\text { dramaturgique : Ramus Heisterberg. }\end{array}$ & $\begin{array}{l}\text { Brouillage entre documentaire et fic- } \\
\text { tion en utilisant les images prises sur le } \\
\text { vif permettant une hybridation du genre } \\
\text { (mise en scène et images du réel se } \\
\text { côtoient dans le même film) : les specta- } \\
\text { teurs se retrouvent à hauteur d'homme, à } \\
\text { pied d'égalité avec les soldats danois. Ils } \\
\text { ne peuvent pas se poser en tant que juge. } \\
\text { Cette technique a été utilisée très large- } \\
\text { ment dans le film Il faut sauver le soldat } \\
\text { Ryan (Spielberg, 1998). }\end{array}$ \\
\hline $\begin{array}{l}\text { Présentation de deux temps-espace (les } \\
\text { spectateurs suivent les soldats avant leur } \\
\text { départ en Afghanistan avec leur famille, } \\
\text { leurs proches mais également sur le ter- } \\
\text { rain en Afghanistan). }\end{array}$ & $\begin{array}{l}\text { Absence de témoignages face caméra et } \\
\text { de la voix-off à noter. }\end{array}$ \\
\hline
\end{tabular}


Dans le film Armadillo, nous retrouvons 1'effet documentaire le plus récent et le plus massif : « la caméra portée, voire agitée, devenue une véritable marotte en cette fin de siècle » (Niney, 2004) accentuant l'allure documentaire d'un film de fiction. «Pourtant la différence subsiste entre les deux genres et d'une façon radicale, car la fiction dans le documentaire sert la réalité, tandis que le documentaire dans la fiction sert la fiction » (Maillot, 2012). En effet, il convient de délimiter la part de la fiction et du documentaire, dans le film Armadillo, tout en prenant acte qu'il existe une zone d'hybridation et une catégorie particulière de film proposant une autre relation au réel. Ne peut-on pas considérer la mise en scène, la dramatisation et la mise en récit comme des effets dramaturgiques utilisés pour rendre le documentaire plus attractif ? Sommes-nous, dans le cas d'Armadillo, face à une nouvelle catégorie générique?

Genre hybride, Armadillo provoque chez le spectateur un brouillage entre la fiction et le documentaire. Ce film propose un rapport à la connaissance et s'inscrit dans l'art de brouiller les lignes de démarcation entre fiction et documentaire. La distinction entre documentaire et fiction est remise en question : « Le pôle de la fiction repose sur l'auteur-réalisateur et le spectateur. Le pôle du documentaire, quant à lui, repose sur l'auteur-réalisateur, le spectateur et le réel » (Maillot, 2012). Nous retrouvons bien ces deux aspects, ces deux pôles bien distincts dans Armadillo (voir tableau 1). En effet, en refusant l'utilisation de la voix-off, des témoignages face caméra, et en intégrant des éléments de mise en scène, Janus Metz invite le spectateur, à l'instar de la plupart des cinéastes contemporains, à orienter son film présenté comme documentaire, aux yeux des spectateurs, vers le cinéma de fiction. Les éléments de marquage indiciel s'entrelacent puisque ensemble, réalisateur et protagonistes, recréent un film fictionnel dans un film documentaire. Le trouble que provoquent les séries, mais aussi la télé-réalité et le documentaire contemporain recouvre un régime où «l'effet de réel » tend à remplacer « l'effet de vérité » (Veyrat-Masson, 2008). En effet, tout en générant des effets de réel, dont l'utilisation de nombreux détails, la mise en scène de l'intimité des personnages ou encore le choix des protagonistes, les différentes images proposées aux spectateurs permettent de générer une valeur d'authenticité aux images.

Dans cette zone de confusion, inhérente à Armadillo, le récepteur n'a qu'accessoirement la promesse d'un genre pour comprendre le genre cinématographique. En proposant aussi aux spectateurs un documentaire sur la guerre, le réalisateur s'érige en héros prenant tous les 
risques (la caméra continue de filmer pendant les scènes de combats) requérant l'adhésion du spectateur. En effet, Janus Metz leur propose sa représentation de la guerre en Afghanistan (le réalisateur danois Janus Metz a souhaité réaliser un film au plus proche de la réalité des nombreux combats menés en Afghanistan. Il a accompagné au quotidien une troupe de soldats danois). Deux techniques, deux approches, participant encore plus au brouillage entre fiction et documentaire et entraînant, chez le spectateur, des émotions bien distinctes. «Le film de fiction peut être plus vrai que le vrai parce que le choc qu'il peut produire sur nous restitue peut-être mieux les chocs qu'on a pu connaitre en histoire qu'un film documentaire plus éloigné » (Ferro, 2005). Janus Metz l'a bien compris et n'hésite pas à entraîner le spectateur dans ce basculement incessant dans plusieurs genres filmiques. Entre choc émotionnel, confusion entre monde réel et monde virtuel (le réalisateur alterne passage des soldats jouant aux jeux-vidéo et scènes réelles de la guerre en Afghanistan), monde de la fiction et de la représentation du réel, le réalisateur du film Armadillo propose une ressemblance des images représentées aussi bien avec celles des jeux-vidéo (le réalisateur propose souvent des plans utilisant les codes visuels des jeux-vidéo), images virtuelles, que celles des représentations du réel et de la fiction. Des images des soldats danois « jouant» à la guerre comme dans un jeu-vidéo, dans un environnement réel, la guerre en Afghanistan, côtoient de nombreux éléments issus aussi bien de la fiction que du documentaire, Armadillo confronte donc le spectateur à ce brouillage continuel.

« C'est une attente du lecteur du texte historique que l'auteur lui propose un récit vrai et non une fiction. La question est ainsi posée de savoir si, comment, et jusqu'à quel point, ce pacte tacite de lecture peut être honoré par l'écriture de l'histoire» (Ricœur, 2000). Les spectateurs attendent, tout comme un lecteur du texte historique avec un auteur, que le réalisateur d'Armadillo leur propose un récit vrai, un état des lieux de la guerre en Afghanistan. Le spectateur n'est-il pas en attente de la présence du réel lorsqu'il visionne un film qui présente, en priorité, les horizons d'attente d'un documentaire?

«La notion de cadre d'interprétation proposé par Goffman, de par sa dimension interprétative et sociologique, semble utile à rapprocher de l'effet du réel » (Glevarec, 2010). En effet, nous privilégions ici les effets sur la réception et le rôle de la croyance à travers l'étude des discours tenus sur le film du point de vue des spectateurs. 


\section{2. Étude des discours tenus sur le film Armadillo}

«Le cinéma noue une ambiguïté avec le réel » (Rosset, 1978). Dans le cadre de notre film Armadillo, nous ne sommes pas face à un phénomène nouveau mais à la continuité d'un fait connaissant une longue histoire au cinéma. En effet, dans Armadillo Janus Metz s'inspire de Werner Herzog, réalisateur et représentant du nouveau cinéma allemand des années 1960-1970, en plaçant des personnages dans des contextes extrêmes afin de nous donner à considérer la fiction comme un documentaire et le documentaire comme une fiction. Effectivement, tout au long de sa carrière, le cinéaste alternera fictions et documentaires, les deux se mêlant et se répondant sans cesse, dans des histoires où les hommes vont jusqu'au bout et même au-delà, comme en témoigne l'un des sommets du versant « fictionnel » Aguirre $^{10}$ (Herzog, 1975).

\subsection{Du point de vue des spectateurs}

L'étude de la réception a comme objectif de compléter les réflexions explicitées dans les pages précédentes. Les critiques des spectateurs, laissées sur les sites internet, peuvent apparaître comme une source de compréhension de ce brouillage entre fiction et représentation du réel. En effet, les avis du public témoignent d'une tout autre réception. Il nous faudra donc comprendre quel rôle a joué leur croyance dans leur perception du film Armadillo. Les spectateurs ont-ils perçu une fiction ou un documentaire? Comment se définit leur horizon d'attente?

D'un point de vue méthodologique, nous nous sommes appuyés sur l'étude des 26 critiques postées sur le site Sens critique ${ }^{11}$ entre le 15 décembre 2010 et le 3 janvier 2018. Nous les avons classées en trois catégories : les commentaires négatifs, positifs et mitigés envers le film. Une fois établi ce premier classement, nous avons distingué les commentaires en fonction de leur niveau d'analyse (sémantique, syntaxique et pragmatique).

$10 \mathrm{Au}$ milieu du XVI $\mathrm{I}^{\mathrm{e}}$ siècle, un groupe de conquérants espagnols venus par la cordillère des Andes pénètre dans la forêt vierge, persuadé d'y trouver l'Eldorado, le pays de l'or raconté par les Incas. Mais l'épuisement, les maladies et les attaques des Indiens contrarient leur expédition.

11 Nous proposons une étude des critiques des spectateurs, sur le film Armadillo, émises sur le site internet Sens critique. Accès : https:/www.senscritique.com/film/ Armadillo/408349/critiques. [Consulté le 25/04/2018]. 
Sur les 26 critiques, quatre éreintent le film. Trois remettent en doute la véracité du film ; leur attente était de visionner un documentaire et ils reprochent le côté télé-réalité voire voyeuriste proposé par le réalisateur : "Armadillo, en plus d'être un mauvais documentaire, ennuyeux, n'apprenant rien, voyeur et esthétiquement laid, reste un summum de lâcheté qui dévalorise plus qu'autre chose le rôle des forces armées », «Un documentaire quasi irréel donc on a droit à tout et c'est bien le problème », «Un pan entier ressemble à de la fiction et nous nous retrouvons dans une position de voyeur $»$.

Une autre critique s'appuie sur le manque de réalisme du film : «On nous montre des scènes clichées, mises bout à bout pour nous faire croire à un réalisme au final totalement absent du film ».

Concernant les critiques positives des spectateurs (au nombre de 22), ils apprécient cette immersion dans le monde réel sous la forme d'un documentaire (6 critiques) : «Une immersion dans la bien triste réalité qu'est celle de la guerre », « Capturée au plus près de l'action avec une prise de risque maximale, la réalité s'offre au spectateur », « Armadillo permet pour la première fois d'être acteur non pas d'une retranscription de la guerre mais de la pure et stricte réalité », « Documentaire vraiment fort », « Documentaire proche de la réalité », « Documentaire hallucinant $\gg$.

D'autres internautes ( 5 critiques) sont conscients de ce brouillage entre réalité et fiction et apprécient cette prise de risque de la part du réalisateur : " Janus Metz dynamite les règles inhérentes au documentaire, en créant une porosité porteuse d'ambigüité entre fiction et réel », « Très fictionnalisé, il n'en demeure pas moins l'une des œuvres les plus réalistes jamais tournées sur la guerre en Afghanistan », « Documentaire de haute volée gâché par un peu trop de fictionnalisation », « La frontière entre le docu et la fiction étant rendue beaucoup plus floue comme le rapport qu' eux ont entre le virtuel et le réel », « Un documentaire qui évolue ».

Un internaute souligne «On oublie que c'est un documentaire, plus fort que Striptease $»$.

Certains internautes utilisent même le terme reportage : « Le reportage est très bien mené », « En sortant de la séance, j'étais persuadé qu'Armadillo était une fiction avec un montage légèrement orienté reportage $[\ldots]$ et puis je me suis renseigné sur Internet et j'ai appris que tout était vrai, y compris les morts $»$.

Notre analyse des discours tenus sur le film Armadillo met au jour plusieurs éléments. En effet, les critiques émises par les internautes sou- 
lignent l'ambiguïé du film Armadillo proposant des caractéristiques d'une certaine esthétique du documentaire tout en soulignant un brouillage intentionnel entre fiction et documentaire. Les critiques émises par les internautes confirment donc qu'Armadillo incarne bien à la fois le monde historique et contextuel du documentaire et le monde fictif suscitant des interrogations de la part des spectateurs en raison de cette hybridation entre fiction et représentation du réel.

Les « doublures du réel » que propose le cinéma du réel contemporain sont polymorphes, se jouant des frontières entre fiction et réalité, abolissant parfois volontairement la séparation entre filmeur et filmé, n'hésitant pas à fissurer la dichotomie entre évidence historique et récit sensible, ou encore lançant des passerelles entre expérimentation plastique et récit filmique (Maury, 2011).

Ce corpus nous rappelle que les spectateurs s'organisent en différents groupes proposant des grilles de lecture différentes selon leur horizon d'attente. Nous avons saisi ici de quelles manières le film Armadillo a été perçu par le public. Il faut être conscient que les spectateurs n'ont pas les cadres réels sur lesquels s'appuyer afin de distinguer la représentation du réel et de la fiction. Le réalisateur est lucide sur ce point et en joue en prenant comme référence la guerre en Afghanistan (authentique : elle existe); c'est donc à l'issue du visionnage que les spectateurs sont dans une perspective de compréhension du quotidien des soldats danois en Afghanistan et sont donc engagés dans des processus de croyance de la situation. Mais, si les films de fiction nous apprennent de l'histoire, par des procédures involontaires de la part du réalisateur, bien souvent, « ils peuvent aussi nous mentir beaucoup plus par le choc qu'ils produisent, que les films documentaires ou même que les écrits » (Ferro, 2005). Janus Metz propose une vision partielle, du côté des soldats danois, de la guerre en Afghanistan. Cette approche fait écho aux propos de Marc Ferro : « Je suis actuellement un peu en guerre contre ce film sur Hitler qui vient de sortir, La Chute, et d'abord en raison du tintamarre autour du film. On présente le film comme faisant le portrait d'un Hitler humain... Allons-nous faire une série : « Staline humain », "Gengis Khan humain... » sans évoquer le reste ? » (Ferro, 2005). 
« Le rôle de la croyance est beaucoup plus déterminant que les savoirs dans le langage cinématographique " (Jost, 2004) ${ }^{12}$. François Niney reprend cette approche à travers la théorie goffmanienne de la modalisation et des cadres d'interprétation pour décrire les régimes de croyance à l'endroit du documentaire et de la fiction ; " il déduit que l'horizon d'attente du premier est la connaissance tandis que celui de la seconde est la crédibilité » (Glevarec, 2009). Cette caractérisation nous parait plus convaincante dans le cadre de notre étude ; nous retrouvons aisément ces deux horizons d'attente dans le film Armadillo : la connaissance car le réalisateur donne à comprendre aux spectateurs le déroulement et les conséquences de la guerre en Afghanistan et la crédibilité car il donne à voir dans son film, grâce à l'utilisation de certains procédés (voir tableau 1) et à la présence des soldats danois, la réalité de cette guerre. Il convient donc de considérer le film Armadillo comme un genre hybride ; à la croisée du docufiction brouillant la fiction avec un champ réel et du documentaire dramatisé. En effet, son film est construit, produit et diffusé comme un documentaire. On y retrouve des éléments de mixité par des effets de spectacularisation. Peut-on parler de scènes rejouées comme le générique final semble l'indiquer avec la présence " d'une directrice de casting » ? Ainsi, il convient de porter un regard disciplinaire sur ce genre, favorisant la réflexion critique et l'acquisition de nouveaux outils de compréhension tout en gardant à l'esprit, comme nous l'avons énoncé précédemment, que le rôle de la croyance est décisif pour le spectateur dans sa perception d'un genre cinématographique.

Mener une réflexion sur ce qui est aujourd'hui appelé docufiction, en tant que genre hybride où fiction et rapport au réel se mêlent de manière à troubler le spectateur dans son contrat de lecture et l'horizon de ses attentes est désormais indispensable (Klein \& Tixhon, 2009).

12 Vidéo d'une conférence de François Jost en 2004. Pour lui, les fluctuations de la réception ont montré que les croyances jouent un rôle bien plus déterminant que les savoirs sur l'image dans son interprétation. Accès : https://www.canalu.tv/video/ univserite_de_tous_les_savoirs/cinema_television_entre_realite_et_fiction.1403 [Consulté le 25/04/2018]. 


\section{Réflexions conclusives}

« L'hybridation, phénomène omniprésent dans de nombreux domaines, a particulièrement influencé l'évolution du cinéma » (Amiard, 2010) ; faut-il y inclure, dans cette approche, le film Armadillo ? L'étude de la réception nous a permis d'observer comment certains spectateurs se réapproprient ce phénomène d'hybridation qui s'attache à estomper les frontières entre réel et virtuel mais aussi entre réalité et fiction. Entre effets de réel, figures majeures de notre contemporanéité, et nouvelle forme d'esthétique cinématographique, Armadillo souligne l'influence de la représentation du réel sur la croyance des spectateurs et plus spécifiquement, dans notre approche, sur la guerre en Afghanistan.

Le trouble est d'autant plus important que dès la bande annonce ( Ce film n'est pas une fiction, ce n'est pas un film de guerre, juste... la réalité »), le spectateur se retrouve dans ce tourbillon de confusion dans lequel il est difficile de définir le genre cinématographique proposé ; le réalisateur ne cherche pas à faire croire aux spectateurs mais va bien au-delà. Il s'attache à susciter le questionnement des spectateurs face à la représentation cinématographique de la guerre en Afghanistan. Janus Metz s'appuie sur un événement appartenant à des faits réels demandant au spectateur une interprétation d'une réalité qui peut toujours être remise en doute. Au-delà d'une simple prétention à l'égard de la question de l'authenticité et du spectateur (Picard, 2018), Armadillo nous invite à repenser la définition de la transcendance du réel. L'utilisation du phénome d'hybridation proposé par le réalisateur souligne le réel comme dimension, comme une pensée de l'expérience vécue par les soldats en Afghanistan. Nous retrouvons ici le sens actif de transcendance, définit par de nombreux penseurs comme Husserl ou Heidegger, pour indiquer un dépassement de soi. Janus Metz plonge les spectateurs au-delà de l'expérience de la guerre. Il propose, par les procédés utilisés, une nouvelle catégorie générique, un nouvel ensemble de pratiques sociales associées à un nouveau regard sur le film de guerre : la conscience des soldats danois. Janus Metz utilise le cinéma comme un outil de transcendance du réel plongeant les spectateurs face à une nouvelle création artistique interrogeant les principes d'une réalité plus vaste et complexe.

Nos propos ont tenté de dégager les différents modes spécifiques à Armadillo et de nous positionner dans une perspective pragmatique, en nous basant sur une étude des discours tenus sur le film Armadillo, car assurément, Armadillo nous questionne et nous interroge. 
Le même programme peut être tiré du côté de la réalité ou du côté de la fiction, selon la connaissance du public de la réalité représentée, d'autre part, son acceptation comme son évaluation dépendent du degré de réalité qu'on lui accorde (Jost, 2010).

Utilisant de nombreux effets de réel, Janus Metz documente à sa façon le réel. Quelles images de la réalité le film Armadillo nous donne-t-il à voir ? Il illustre des faits de guerre et propose aux spectateurs de réfléchir aux différents enjeux politiques liés à cette guerre. Nous sommes depuis longtemps dans un rapport de spectacularisation du monde sur laquelle se calque notre perception. « Ce qui compte est l'efficacité spectaculaire, mais quand ces dimensions sont vraiment saisies sur le vif, on entre dans une certaine confusion » (Comolli, 2008). Armadillo engendre, plus spécifiquement, de la part des spectateurs une recherche de la valeur historique des images diffusées et une quête de la vérité par rapport à la translittération de l'évènement, dans notre cas, la guerre en Afghanistan. Quelle part d'imaginaire recouvre cette réalité ? $\mathrm{Au}$-delà de ce métissage proposé par Janus Metz, le réalisateur propose au public une nouvelle relation au réel voire une transformation du réel. En effet, même si les faits représentés appartiennent à un passé proche, il convient d'apporter une réflexion constructive sur le traitement futur accordé à ce film qui, nous le rappelons, se définit, d'après la bandeannonce, comme la réalité. Cette relation au réel est accentuée car, dans notre contexte actuel, elle apparaît comme un moyen d'attirer le plus d'audience ; la représentation de la réalité fait vendre et attire le public.

Armadillo participe d'un courant général dont on peut interroger les principes, voire les critiquer, mais sûrement pas les ignorer. En effet, la distinction originelle entre le documentaire et la fiction n'est donc pas infondée mais elle est ici remise en question. Aussi cette hybridation, entre représentation du réel et fiction, ne conduit-elle pas le spectateur à dépersonnaliser l'action de ces jeunes soldats danois (acteurs d'une guerre par nature brutale, concrète et impersonnelle) face à l'intensité de cette guerre? 


\section{Interviews}

Entretien face à face avec François Garçon, Paris, le 5 janvier 2011.

\section{Filmographie}

Aguirre, 1975, Werner Herzog, Allemagne. Armadillo, 2010, Janus Metz, Danemark.

Il faut sauver le soldat Ryan, 1998, Steven Spielberg, États-Unis.

La Chute, 2005, Olivier Hirschbiegel, Allemagne.

Le projet Blair Witch, 1999, Daniel Myrick et Eduardo Sanchez, États-Unis.

\section{Références}

Ambroise-Rendu, A.C. \& Veyrat-Masson, I. (2005). Entretien avec Marc Ferro : guerre et images de guerre. Le Temps des médias 4, 239-251.

Amiard, J.-F. (2010), L 'hybridation, de nouvelles formes cinématographiques amenées par les dernières technologies dans l'esthétique des films. Thèse de doctorat en recherches cinématographiques et audiovisuelles, sous la direction de Gérard Leblanc, Université Sorbonne Nouvelle, 560 p.

Comolli, J.-L. (2008). Mauvaises fréquentations : document et spectacle. Revue Images documentaires $63,1^{\mathrm{er}}$ et $2^{\mathrm{e}}$ trimestres.

Frodon, J.-M. (1998). La Projection nationale, cinéma et nation. Paris : Éditions Odile Jacob, $248 \mathrm{p}$.

Garçon, F. (2006). Enquête sur le cauchemar de Darwin. Paris : Flammarion, 265 p.

Glevarec, H. (2009). François Niney, Le documentaire et ses faux semblants. Histoire des médias [En ligne].

Glevarec, H. (2010). Trouble dans la fiction. Effets de réel dans les séries télévisées contemporaines et post-télévision. Questions de communication 18, 214-238.

Jost, F. (1997). La promesse des genres. Réseaux 15(81). Dossier Le genre télévisuel. pp. 11-31.

Jost, F. (2010). Que signifie parler de réalité ? Revue Télévision 1, Dossier Télévision et réalité, CNRS Éditions, 208 p.

Klein, A. \& Tixhon, A. (2009). La Communication audio-visuelle : entre réalité et fiction : approche pluridisciplinaire. Namur : Presses universitaires de Namur, $170 \mathrm{p}$.

Maillot, P. (2012). L'écriture cinématographique de la sociologie filmique. Comment penser en sociologue avec une caméra ? La Nouvelle Revue du travail [En ligne], 1, mis en ligne le 10 décembre 2012.

Maury, C. (2011). Habiter le monde : éloge du poétique dans le cinéma du réel (Coll. Côté cinéma). Crisnée : Éditions Yellow Now, 188 p.

Metz, C. (1977). Le Signifiant imaginaire : psychanalyse et cinéma. Choix essais, 370 p. Niney, F. (2009). Le Documentaire et ses faux-semblants, Paris : Klincksieck, 208 p.

Niney, F. (2004). L'Épreuve du réel à l'écran, Bruxelles: De Boeck, 340 p.

Picard, A. (2018). Qu'est-ce que le réel ? Des cinéastes prennent position. Fécamp : Post Éditions, 352 p. 
Ricœur, P. (2000). L'écriture de l'histoire et la représentation du passé. Annales. Histoire, Sciences Sociales 55(4), 731-747.

Rivette, J. Cahiers du Cinéma.

Rosset, C. (1978). Le Réel. Traité de l'idiotie (Coll. critique). Paris : Éditions de Minuit, $160 \mathrm{p}$.

Veyrat-Masson, I. (2008). Télévision et histoire, la confusion des genres : Docudramas, docufictions et fictions du réel, Bruxelles : De Boeck. 


\section{(c) (i) $\left(\Theta_{\mathrm{BY}} \mathrm{NC}_{\text {PD }}\right.$ Publié sous la licence Creative Common}

«Attibution - pas d'utilisation Commerciale - Pas de Modification 4.0. International» (CC BY-NC-ND) 